\title{
$\mathrm{Cl}$ i ni copat hol ogi cal si gni fi cance of ant i nucl ear ant i bodi es i $n$ non- al cohol i $c$ st eat ohepat i t i s
}

\begin{tabular}{|l|l|}
\hline 著者 & $\begin{array}{l}\text { N wa H deki, Sasaki Mot oko, Har at ake Joj i , } \\
\text { Kasai Takahi ko, Kat ayanagi Kazuyoshi, Kur unaya } \\
\text { H roshi, Mat suda Shi nj i, M nat o H r oshi, Zen } \\
\text { Yoh, Uchi yana Aki o, M wa At suo, Sai to } \\
\text { Kat suhi ko, Sudo Yoshi ko, Nakanuma Yasuni }\end{array}$ \\
\hline $\begin{array}{l}\text { j our nal or } \\
\text { publ i cat i on ti t l e }\end{array}$ & Hepat ol ogy Resear ch \\
\hline vol une & 37 \\
\hline number & 11 \\
\hline page r ange & $923-931$ \\
\hline year & $2007-11-01$ \\
\hline URL & ht t p: //hdl . handl e. net /2297/7120 \\
\hline
\end{tabular}




\section{Clinicopathological significance of antinuclear antibodies in non-alcoholic steatohepatitis}

Hideki Niwa ${ }^{1}$, Motoko Sasaki ${ }^{1}$, Joji Haratake ${ }^{2}$, Takahiko Kasai ${ }^{3}$, Kazuyoshi Katayanagi ${ }^{4}$, Hiroshi Kurumaya ${ }^{4}$, Shinji Masuda ${ }^{5}$, Hiroshi Minato ${ }^{6}$, You Zen ${ }^{6}$, Akio Uchiyama ${ }^{7}$, Atsuo Miwa $^{7}$, Katsuhiko Saito ${ }^{8}$, Yoshiko Sudo ${ }^{9}$, Yasuni Nakanuma ${ }^{1}$

1. Department of Human Pathology, Kanazawa University Graduate School of Medicine, Kanazawa, Japan. 2. Department of Pathology, Kurobe City Hospital, Kurobe, Japan.

3. Department of Diagnostic Pathology, Nara Medical University, Nara, Japan.

4. Department of Pathology, Ishikawa Prefectural Central Hospital, Kanazawa, Japan.

5. Department of Pathology, Kouseiren Takaoka Hospital, Takaoka, Japan. 6. Pathology

Section, Kanazawa University Hospital, Kanazawa, Japan. 7. Department of Pathology, Toyama Prefectural Central Hospital, Toyama, Japan. 8. Department of Pathology, Toyama City Hospital, Toyama, Japan. 9. Department of Pathology, Fukui Saiseikai Hospital, Fukui, Japan

A short running title: Clinicopathological significance of ANA in NASH patients Corresponding author: Motoko Sasaki, MD. PhD

Department of Human Pathology, Kanazawa University Graduate School of Medicine, Takaramachi13-1, Kanazawa 920-8640, JAPAN

TEL:+81-76-265-2197 FAX: +81-76-234-4229

Email: m8sasaki@med.kanazawa-u.ac.jp 


\begin{abstract}
Serum antinuclear antibodies (ANA) are occasionally noted in the patients with non-alcoholic steatohepatitis (NASH). We examined the significance of ANA in NASH by comparing the clinicopathological features in the patients with ANA-positive NASH $(n=35)$ and ANA-negative NASH ( $n=36)$. Inflammatory cell profiles and the distribution of oxidative stress markers were also examined immunohistochemically. The ANA-positive NASH was significantly associated with female gender $(p=.005)$, high degree of portal inflammation $(\mathrm{p}=.039)$, interface activity $(\mathrm{p}=.036)$ and hepatocellular ballooning $(\mathrm{p}=.0008)$. In addition, The ANA of high-titer (320-fold or more) was significantly associated with the histological grade and stage of NASH $(p=.02)$. The degree of steatosis is rather mild in high-titer ANA group $(\mathrm{p}=.01)$. The analysis of inflammatory cell profiles revealed that CD3-positive T cells were predominant and plasma cells were rather few in portal area and hepatic lobules in both ANA-positive and ANA-negative groups. There was no difference in the distribution of oxidative stress markers between ANA-positive and ANA-negative groups. These findings suggest that the presence of ANA may be related to the progression of NASH and that a different type of autoimmune mechanisms may be involved in the pathogenesis of NASH with ANA, compared to the pathogenesis of autoimmune hepatitis.
\end{abstract}

Key Words; nonalcoholic steatohepatitis, antinuclear antibody, portal inflammation, autoimmunity 


\section{Introduction}

Nonalcoholic fatty liver disease (NAFLD) becomes a recognized clinical entity, associated with obesity, diabetes mellitus and other excess nutrition uptake ${ }^{1}$. Nonalcoholic steatohepatitis (NASH) is a part of NAFLD and characterized by histological features resembling alcoholic hepatitis such as steatosis, hepatocellular ballooning, neutrophilic infiltration and fibrosis ${ }^{2,3}$. Recently, NASH is recognized as a cause of cryptogenic cirrhosis and hepatocellular carcinoma ${ }^{4,5}$. The pathogenesis of NASH is multifactorial and the two hits theory is well known ${ }^{6}$, which accounts for accumulation of fat as the first hit and hepatocellular injury in fatty liver as the second hit. The oxidative stress, ATP depletion and mitochondrial dysfunction as second hits are suggested to play an important role in hepatocellular injury for the progression of steatohepatits ${ }^{1}$. Insulin resistance is also germane to NASH.

In patients with non-alcoholic fatty liver disease (NAFLD), the presence of autoantibodies, especially antinuclear antibodies (ANA), is noted occasionally. The prevalence of ANA in patients with NAFLD is reportedly higher than the general population, ranging from $12 \%$ to $46 \%{ }^{4,7-11}$. Since ANA is a characteristic parameter in autoimmune hepatitis (AIH), it is critical to differentiate NAFLD with ANA and AIH. There have been several reports about the significance of ANA in NAFLD patient ${ }^{1,8,9,13-16}$, but it remains controversial ${ }^{1,16}$. For example, Adams et al. reported that the positive autoantibodies were associated with higher fibrosis stage, higher inflammatory grade and higher levels of gammaglobulin ${ }^{8}$. In contrast, Cotler et al. reported that ANA in the patients with NASH was not associated with the degree of inflammation, and that it was 
nonspecific antibody response ${ }^{13}$.

In this study, we examined the clinicopathological significance of ANA in NASH, comparing ANA positive cases and ANA negative cases. We put a special emphasis on the histopathological features, the inflammatory cell profile and the distribution of oxidative stress marker.

\section{Materials and Methods}

\section{Subjects}

The study included 35 patients with ANA-positive NASH and 36 with ANA-negative NASH. Although there are many women in the general population who are positive for ANA and low-titer (40-fold) of ANA may be less important in general clinical practice, we regarded ANA-positive when the titer of ANA was 40-fold or more according to the AIH scoring system in which 40 -fold of ANA is scored as +1 . When the titer of ANA was 320-fold or more, ANA was regarded as high-titer. All of these cases were collected from the files of the Department of Human Pathology, Kanazawa University Graduate School of Medicine and affiliated hospitals between 1996 and 2006. All patients were negative for hepatitis viral markers, and there was no history of alcohol abuse. All patients underwent clinical and laboratory evaluation, including AST, ALT, ALP, $\gamma$-GTP and IgG. The diagnosis as NASH was made based on clinical data and pathologic findings of liver biopsy. The patients were classified using AIH scoring system reported by the International Autoimmune Hepatitis Group ${ }^{17}$. The patients with NASH who could be classified as “definite AIH” were not included in this study. 


\section{Liver Biopsy and histopathological evaluation}

One specimen was a wedge biopsy material and the remainings were needle biopsies. Liver biopsy material were fixed in neutral formalin, embedded in paraffin and cut into $4 \mu \mathrm{m}$ sections. Several of them were stained with hematoxylin and eosin and silver reticulin stain. Two liver pathologists examined specimens, who were blind to the titer of ANA. The grade and stage of NASH were evaluated according to the classification of Brunt et al ${ }^{2}$. The degree of steatosis was subclassified as $0,<5 \% ; 1,5-33 \% ; 2,33-66 \%$; 3 , $>66 \%$. The degree of portal inflammation and hepatocellular ballooning was subclassified as 1, mild; 2, moderate; 3, severe. The presence of Mallory body and/or cytoplasmic coagulum was also evaluated with the aid of immunostaining for cytokeratin (CK) 8. The degree of interface activity was evaluated as follows: 0 , none; 1 , minimal; 2 , mild; 3 , moderate to severe.

\section{Immunohistochemistry}

For the assessment of inflammatory cell profile and for the detection of oxidative stress marker, immunohistochemical staining was performed as described previously ${ }^{18}$ using a Ventana automated stainer (Ventana medical systems, Tucson, AZ, USA). Primary antibodies included CD3 (mouse monoclonal (mono); no dilution; Ventana, Tucson, AZ, USA), CD8 (mono, 1/50, Ventana), CD20 (mono, no dilution, Ventana), CD138 (mono; 1/50; Dako, Glostrup, Denmark), myeloperoxidase (MPO)(rabbit polyclonal; 1/50; Dako), CK8 (mono; 1/50; Dako), 4-hydroxy-2'-nonenal (4-HNE)(mono; 1/20; Japanese Aging Control Institute (JAICA)), 4-hydroxy-2-hexenal (4-HHE)(mono; JAICA; 1/500) and 
8-hydroxydeoxyguanosine (8-OHdG) (mono; JAICA; 1/100). Immunostaining for 8-hydroxydeoxyguanosine (8-OHdG) was performed using Ventana Alkaline Phosphatase Enhanced Detection kit and Ventana Basic DAB Detection kit was used with other antibodies. Negative controls included substituting the primary antibody with similarly diluted normal mouse or rabbit immunoglobulin.

Inflammatory cell profile Inflammatory cells positive for CD3 (a marker for T cell), CD8 (cytotoxic/suppressor T cell), CD20 (B cell), CD138 (plasma cell) and myeloperoxidase (MPO) (neutrophil and macrophage) were evaluated in 15 liver specimens of ANA-positive NASH and 15 of ANA-negative NASH. CD8-posiive cells were also assessed in 8 of ANA-positive NASH and 8 of ANA-negative NASH. CD3, CD8, CD20, CD138 and MPO positive cells were counted in 2 and 10 different high power fields (HPF, 10x eyepiece and 40x lens) in the portal area and the hepatic lobule, respectively. Since CD3-positive T cells were predominantly seen in preliminary study, the ratio of CD8 /CD3, CD20/CD3, CD138/CD3 and MPO/CD3 positive cells were evaluated to assess the inflammatory cell profiles in ANA-positive and ANA-negative NASH. In addition, we examined 10 specimens taken from the patients with definite AIH for comparison.

Oxidative stress marker

4-hydroxy-2'-nonenal (4-HNE), 8-hydroxydeoxyguanosine (8-OHdG) and 4-hydroxy2-hexenal (4-HHE) were evaluated as oxidative stress markers ${ }^{19-21}$. Four histological normal livers were also examined as controls. In normal liver samples, there were no positive signals of 8-OHdG, 4-HNE and HHE. For semi-quantitative assessment, 8-OHdG 
or 4-HNE positive hepatocytes were counted at 10 different high power fields (HPF, 10x eyepiece and 40x lens). At least 1000 hepatocytes were examined and the positive rate was expressed as \% of hepatocytes. The degree of 4-HHE immunohistochemical staining was evaluated as follows: 0 ; negative, 1 ; slightly positive, 2; mildly moderately positive, 3; strongly positive.

\section{Statistics}

Data are expressed as the mean \pm SD. Statistical analysis was performed using Mann-Whitney's U test. When p value was less than 0.05 , the difference was regarded as statistically significant.

\section{Results}

\section{Clinical Features in ANA-positive and ANA-negative NASH}

The clinical features and laboratory data are summarized in Table 1. The titer of ANA was ranged 40 to 5120-fold in ANA-positive group (40-fold, n=11; 80-fold, n= 3; 160-fold, n= 10; 320-fold, n=2; 640-fold, n=5; 1280-fold, n= 3; 5120-fold, n= 1). Fourteen and one patients had an AIH score of 10-15 and belonged to probable cases before biopsy in ANA-positive and ANA-negative groups, respectively ${ }^{17}$ Female patients were significantly predominant in ANA-positive group, when compared with ANA-negative group $(p=0.005)$. There was no difference in age, the prevalence of obesity, diabetes mellitus and hyperlipidemia between the ANA-positive and ANA-negative groups. In laboratory data, the level of AST in high-titer (320-fold or more) ANA-positive group was significantly 
high, when compared with the ANA-negative group.

\section{Histological Features in ANA-positive and ANA-negative NASH}

The histological features are summarized in Table 3. Figure 1 showed the example of histopathological findings in ANA-positive and ANA-negative NASH. When ANA-positive NASH and ANA-negative NASH were compared, the degree of hepatocytes ballooning ( $p=0.0008)$, portal inflammation $(p=0.039)$ and interface activity $(p=0.036)$ were significantly high in ANA-positive patients. However, portal inflammation and interface activity was rather mild in ANA-positive NASH, when compared with typical AIH. Lymph follicle formation was not seen and plasma cell infiltration was not evident in ANA-positive and ANA-negative NASH. When histological features were included in AIH scoring system $^{17}$ after liver biopsy, 4 patients had AIH score of 10 or 11 in ANA-positive group. Although grade of NASH tended to be higher in ANA-positive patients, there were no significant differences. When the patients with NASH with high-titer ANA were compared with ANA-negative NASH patients, histological grade $(\mathrm{p}=0.02)$ and stage of NASH ( $p=0.02)$, portal inflammation $(p=0.006)$, interface activity $(p=0.0001)$ and hepatocellular ballooning $(\mathrm{p}=0.03)$ were significantly high in high-titer ANA with NASH. The degree of steatosis was rather low in high-titer ANA-positive NASH, when compared with ANA-negative NASH ( $\mathrm{p}=0.02)$. There was no significant difference in the degree of lobular inflammation and the presence of cytoplasmic coagulum between high-titer ANA-positive NASH and ANA-negative NASH. 


\section{Inflammatory cell profiles in ANA-positive and ANA-negative NASH}

The inflammatory cell profile in portal area and hepatic lobules is summarized in Table 3 and 4, respectively. CD3 positive T cells were predominant in portal area and hepatic lobules in both of ANA-positive and ANA-negative NASH (Fig.2). CD8-positive cells are about a half of CD3-positive cells in number. CD3 and CD8-positive cells were seen at the interface of portal area and hepatic lobules. CD138-positive plasma cells and MPO-positive neutrophils/macrophages were occasionally seen in portal area and hepatic lobules. There were no significant differences in the inflammatory cell profiles in portal tracts and hepatic lobule between ANA-positive and ANA-negative patients. CD20/ CD3 and CD138/CD3 ratios were significantly low in portal area in ANA-positive and ANA-negative NASH, when compared with those in AIH $(\mathrm{p}<0.05)$. CD8/ CD3 ratio was rather high in portal area in ANA-negative NASH, when compared with those in AIH $(\mathrm{p}<0.05)$ (table 3). MPO/CD3 ratio was significantly high in hepatic lobules in ANA-positive and ANA-negative NASH, when compared with those in AIH $(\mathrm{p}<0.05)$. Whereas, CD138/CD3 ratio was significantly low in hepatic lobules in ANA-positive and ANA-negative NASH compared with those in AIH $(\mathrm{p}<0.05)$ (table 4).

\section{Oxidative stress markers in ANA-positive and ANA-negative NASH}

The distribution of oxidative stress markers is summarized in Table 6. In accordance with

previous reports $^{21-23}$, the expression of 8-OHdG was detected in the nuclei of hepatocytes, sinusoidal cells and some inflammatory cells (Fig 3). 8-OHdG was rather predominantly seen in hepatocytes in the centrilobular area. 4-HNE was detected in the granules in the 
cytoplasm of hepatocytes (Fig 3) and 4-HHE was detected diffusely in the cytoplasm of hepatocytes to various degrees. There were no significant differences between ANA-positive and ANA-negative NASH in the distribution of 8-OHdG and 4-HNE.

\section{Discussion}

ANA is occasionally detected in patients with NASH and its significance of ANA remains

controversial, so far ${ }^{1,4,7-16}$. To address this issue, we compared clinical and pathological features in the patients with ANA-positive and ANA-negative NASH in this study. In the present study, ANA-positive NASH was associated with female gender. This female predominance agreed with the previous study reported by Cotler et al. ${ }^{13}$ The patients were rather old and the prevalence of obesity, diabetes mellitus and hyperlipidemia were slightly higher in ANA-positive NASH, but the difference was not significant. There have been no previous studies reporting the association of these factors with ANA-positive NASH. Regarding insulin resistance, Loria et al. reported that high-titer ANA was associated with insulin resistance ${ }^{9}$, whereas Adams et al. reported that it was neither associated with higher fasting insulin levels or insulin resistance ${ }^{11}$. Since the present study is retrospective one, details clinical data regarding insulin resistance was not available.

The detail histological evaluation in the present study revealed that ANA-positive NASH was significantly associated with high degree of portal inflammation, interface activity and hepatocellular ballooning. Furthermore, it is of interest that ANA of high-titer (320-fold or more) with NASH was significantly associated with the histological grade and stage of NASH, the degree of portal inflammation and interface activity. The degree of 
steatosis is rather mild in high-titer ANA group. The findings in the present study support the previous study ${ }^{8}$ reporting the association of ANA in NASH with higher fibrotic stage and necroinflammatory grade. Taken them into consideration, it is plausible that the high-titer of ANA may be related to the progression of NASH. There have been no reports describing the higher degree of hepatocellular ballooning in ANA-positive patients, so far. The present study reported firstly the association of hepatocellular ballooning with the presence of ANA.

Although the combination of ANA-positive, high degree of portal inflammation and interface activity may suggest autoimmune features, the overlap of AIH appears to be unlikely in our ANA-positive NASH. Portal inflammation and interface activity were rather mild, when present, compared to typical AIH. Lymph follicle formation and plasma cell infiltration were not evident and the findings were insufficient to make a diagnosis as AIH. When histological features were included in AIH scoring system ${ }^{17}$ after liver biopsy, 4 patients had AIH score of 10 or 11 in ANA-positive group. The inflammatory cell profiles revealed that the infiltration of CD3-positive T cells was predominant in portal area. It is of interest that CD20/ CD3 and CD138/CD3 ratios were significantly low in portal area in ANA-positive and ANA-negative NASH, when compared with those in AIH. MPO/CD3 ratio was significantly high in hepatic lobules in ANA-positive and ANA-negative NASH, when compared with those in AIH. Whereas, CD138/CD3 ratio was significantly low in hepatic lobules in ANA-positive and ANA-negative NASH compared with those in AIH. These data clearly exclude the possible overlap of AIH, irrespective of rather high degree of portal inflammation and interface activity in ANA-positive NASH. Furthermore, these 
differences of inflammatory cell profiles between ANA-positive NASH and AIH suggest that a different type of autoimmune mechanisms may be involved in the pathogenesis of ANA-positive NASH and related to the progression of NASH.

Recently, Albano et al. ${ }^{24}$ reported that circulating IgG against lipid peroxidation products including malondialdehyde (MDA) was significantly higher in NAFLD patients than in controls. Oxidative stress-dependent immune responses were not associated with obesity, type 2 diabetes, or with serum cholesterol, ferritin, or aminotransferase levels ${ }^{24}$. Titers of lipid peroxidation related antibodies were also independent of the extent of steatosis and were similarly distributed in patients with and without necroinflammation ${ }^{24}$. In contrast, lipid peroxidation related antibodies were significantly increased in patients with advanced fibrosis or cirrhosis ${ }^{24}$. These results indicate that the presence of immune reactions triggered by oxidative stress can be an independent predictor of progression of NAFLD to advanced fibrosis ${ }^{24}$. In the present study, the oxidative stress markers (8-OHdG, 4-HNE and 4-HHE) were frequently detected in both ANA-positive and ANA-negative cases, suggesting the oxidative stress was involved in the pathogenesis of NASH. Since histological grade and stage of NASH was high in high-titer ANA-positive NASH group in this study, immune response towards lipid peroxidation in the patients with NAFLD might be related to the generation of autoantibodies. Further analysis is demanded to clarify this point.

Similarly to NAFLD, the presence of non-organ specific antibodies (NOSAs) including ANA has been reported in HCV-related chronic liver disease $\mathrm{e}^{25-27}$. The association between the presence of NOSAs and the clinical, biochemical, and histological picture of 
HCV related chronic liver disease is still controversial ${ }^{25,26}$. Several studies reported a higher biochemical and histological activity in patients with autoantibodies and with HCV related chronic liver disease $\mathrm{e}^{25,26}$. Interestingly, the presence of autoantibodies correlated with the activity of liver disease, suggesting a hypothetical role in the progression of liver damage ${ }^{25,26}$. Furthermore, a study reported that ANA-positive chronic hepatitis C showed poorer response to IFN therapy ${ }^{29}$. In contrast, other reports have failed to identify the presence of NOSAs as an untoward factor for chronic liver disease ${ }^{27,28}$. Autoantibodies in the patients with HCV-related chronic liver disease may reflect autoimmune reactions associated with viral infection ${ }^{24}$ as described in various viral disorders ${ }^{30-32}$. Alternatively, positive ANA might be associated with the progression of chronic liver disease in viral hepatitis and NASH in common.

In summary, the patients with ANA-positive NASH were characterized by female predominance, higher degree of portal inflammation, interface activity and hepatocellular ballooning. In addition, high-titer ANA was associated with the higher grade and stage of NASH. Inflammatory cell profile in ANA-positive NASH was different from a typical AIH. These findings suggest that the presence of ANA may be related to the progression of NASH and that a different type of autoimmune mechanisms may be involved in the pathogenesis of NASH with ANA, compared to the pathogenesis of AIH. 


\section{References}

1. Neuschwander-Tetri BA, Caldwell SH. Nonalcoholic steatohepatitis: summary of an AASLD Single Topic Conference. Hepatology 2003; 37: 1202-19.

2. Brunt EM, Janney CG, Di Bisceglie AM, Neuschwander-Tetri BA, Bacon BR. Nonalcoholic steatohepatitis: a proposal for grading and staging the histological lesions. Am J Gastroenterol 1999; 94: 2467-74.

3. Ludwig J, Viggiano TR, McGill DB, Oh BJ. Nonalcoholic steatohepatitis: Mayo Clinic experiences with a hitherto unnamed disease. Mayo Clin Proc 1980; 55: 434-8.

4. Caldwell SH, Oelsner DH, Iezzoni JC, Hespenheide EE, Battle EH, Driscoll CJ. Cryptogenic cirrhosis: clinical characterization and risk factors for underlying disease. Hepatology 1999; 29: 664-9.

5. Zen Y, Katayanagi K, Tsuneyama K, Harada K, Araki I, Nakanuma Y. Hepatocellular carcinoma arising in non-alcoholic steatohepatitis. Pathol Int. 2001; 51:127-31.

6. $\quad$ Day CP, James OF. Steatohepatitis: a tale of two "hits"? Gastroenterology 1998; 114: $842-5$.

7. Bacon BR, Farahvash MJ, Janney CG, Neuschwander-Tetri BA. Nonalcoholic 
steatohepatitis: an expanded clinical entity. Gastroenterology 1994; 107: 1103-9.

8. Adams LA, Lindor KD, Angulo P. The prevalence of autoantibodies and autoimmune hepatitis in patients with nonalcoholic fatty liver disease. Am J Gastroenterol 2004; 99: 1316-20.

9. Loria P, Lonardo A, Leonardi F, et al. Non-organ-specific autoantibodies in nonalcoholic fatty liver disease: prevalence and correlates. Dig Dis Sci 2003; 48: 2173-81.

10. Laroussi N, Mosnier JF, Morel Y, Deugnier Y, Dumas O, Audigier JC. Non alcoholic steatohepatitis: a multifactorial, frequent, paucysymptomatic liver disease with a fibrotic outcome. Gastroenterol Clin Biol 2002; 26: 475-9.

11. Loria P, Carulli N, Lonardo A. The prevalence of autoantibodies and autoimmune hepatitis in patients with nonalcoholic fatty liver disease. Am J Gastroenterol 2005; 100: 1200-1; author reply 1201-2.

12. Yatsuji S, Hashimoto E, Kaneda H, Taniai M, Tokushige K, Shiratori K. Diagnosing autoimmune hepatitis in nonalcoholic fatty liver disease: is the International Autoimmune Hepatitis Group scoring system useful? J Gastroenterol 2005; 40: 1130-8.

13. Cotler SJ, Kanji K, Keshavarzian A, Jensen DM, Jakate S. Prevalence and 
significance of autoantibodies in patients with non-alcoholic steatohepatitis. J Clin Gastroenterol 2004; 38: 801-4.

14. Tajiri K, Takenawa H, Yamaoka K, Yamane M, Marumo F, Sato C. Nonalcoholic steatohepatitis masquerading as autoimmune hepatitis. J Clin Gastroenterol 1997; 25: 538-40.

15. Czaja AJ, Homburger HA. Autoantibodies in liver disease. Gastroenterology 2001; 120: $239-49$.

16. Sanyal AJ. AGA technical review on nonalcoholic fatty liver disease. Gastroenterology 2002; 123: 1705-25.

17. Alvarez F, Berg PA, Bianchi FB, et al. International Autoimmune Hepatitis Group Report: review of criteria for diagnosis of autoimmune hepatitis. J Hepatol 1999; 31: 929-38.

18. Sasaki M, Ikeda H, Sato Y, Nakanuma Y. Decreased expression of bmi1 is closely associated with cellular senescence in small bile ducts in primary biliary cirrhosis. Am J Pathol 2006;169: 831-45

19. Kasai H. Analysis of a form of oxidative DNA damage, 8-hydroxy-2'- 
deoxyguanosine, as a marker of cellular oxidative stress during carcinogenesis. Mutat Res 1997; 387: 147-63.

20. Paradis V, Mathurin P, Kollinger M, et al. In situ detection of lipid peroxidation in chronic hepatitis C: correlation with pathological features. J Clin Pathol 1997; 50: 401-6.

21. Saeki T, Ichiba M, Tanabe N, et al. Expression of oxidative stress-related molecules in circulating leukocytes and urine in patients with chronic viral hepatitis. Liver Int 2006; 26: $157-65$.

22. Seki S, Kitada T, Yamada T, Sakaguchi H, Nakatani K, Wakasa K. In situ detection of lipid peroxidation and oxidative DNA damage in non-alcoholic fatty liver diseases. $J$ Hepatol 2002; 37: 56-62.

23. Seki S, Kitada T, Sakaguchi H. Clinicopathological significance of oxidative cellular damage in non-alcoholic fatty liver diseases. Hepatol Res 2005; 33: 132-4.

24. Albano E, Mottaran E, Vidali M, et al. Immune response towards lipid peroxidation products as a predictor of progression of non-alcoholic fatty liver disease to advanced fibrosis. Gut 2005; 54: 987-93.

25. Cassani F, Cataleta M, Valentini P, et al. Serum autoantibodies in chronic hepatitis 
C: comparison with autoimmune hepatitis and impact on the disease profile. Hepatology 1997; 26: 561-6.

26. Lenzi M, Bellentani S, Saccoccio G, et al. Prevalence of non-organ-specific autoantibodies and chronic liver disease in the general population: a nested case-control study of the Dionysos cohort. Gut 1999; 45: 435-41.

27. Czaja AJ, Carpenter HA, Santrach PJ, Moore SB, Taswell HF, Homburger HA. Evidence against hepatitis viruses as important causes of severe autoimmune hepatitis in the United States. J Hepatol 1993; 18: 342-52.

28. Pawlotsky JM, Ben Yahia M, Andre C, et al. Immunological disorders in C virus chronic active hepatitis: a prospective case-control study. Hepatology 1994; 19: 841-8.

29. Gatselis NK, Georgiadou SP, Tassoppoulos N, et al. Impact of parietal cell autoantibodies and non-organ-specific autoantibodies on the treatment outcome of patients with hepatitis C virus infection: a pilot study. World J Gastroenterol 2005, 11: 482-487.

30. Holborow EJ, Hemsted EH, Mead SV. Smooth muscle autoantibodies in infectious mononucleosis. BMJ1973; 3: 323-325.

31. Pedersen JS, Toh BH, Mackay IR, et al. Segregation of autoantibody to cytoskeletal 
Niwa-19

filaments, actin and intermediate filaments with two types of chronic active hepatitis. Clin Exp Immunol1982; 48: 527-532.

32. Cassani F, Tremolada F, Baffoni L, et al. Markers of "viral" autoimmunity in chronic non-A, non-B post-transfusion hepatitis. Ital J Gastroenterol 1988; 20: 171-174. 


\section{Figure legends}

Fig 1. A) and B) Histopathological findings of ANA-positive NASH. A) Lymphocytic infiltration was seen in the portal tract and fibrous septa. Steatosis was seen in hepatic parenchyma. B) Arrows indicate the lymphocytic infiltration at the edge of the portal tract (interface activity). This specimen was scored as portal inflammation, 3 and interface activity, 2. Hepatocellular ballooning was also seen (arrowheads).

C) and D) Histopathological findings of ANA-positive NASH. C) Lymphocytic infiltration was seen in the portal tract and fibrous septa. Steatosis was seen in hepatic parenchyma. D) There were few lymphocytes in portal tracts. P, portal tract. Hematoxylin and eosin, A and C, x200; B and D, x400 (original magnification)

Fig 2. Inflammatory cell profile in portal area of ANA-positive NASH. A) CD3-positive cells were predominant in the portal tract. Immunostaining for CD3 and hematoxylin. B) CD8-positive cells were about a half of CD3-positive cells in the portal tracts. Immunostaining for CD8 and hematoxylin. C) Compared with T cells, CD20-positive T cells were rather small in number. Immunostaining for CD20 and hematoxylin. D) There were few CD138-positive plasma cells in portal tracts. Immunostaining for CD138 and hematoxylin. P, portal tract. A-D, x400 (original magnification)

Fig 3. The expression of oxidative stress markers. A) 8-OHdG was detected in the nuclei of hepatocytes (arrows). Immunostaining for CD138 and hematoxylin. B) 4-HNE was detected in granules in the cytoplasm of hepatocytes. Immunostaining for 4-HNE and 
Niwa-21

hematoxylin. (c) HHE was detected in fine vesicles in the cytoplasm of hepatocytes. Immunostaining for HHE and hematoxylin. A-C, x400 (original magnification). 
Table 1 Clinical features in the patients with non-alcoholic steatohepatitis with and without serum antinuclear antibody

\begin{tabular}{|c|c|c|c|c|c|}
\hline & $\begin{array}{l}\text { ANA-negative NASH } \\
(\mathrm{n}=36)\end{array}$ & $\begin{array}{c}\text { ANA-positive NASH } \\
(\mathrm{n}=35)\end{array}$ & p value* & $\begin{array}{l}\text { High-titer ANA NASH } \\
\qquad(\mathrm{n}=11)\end{array}$ & p value* \\
\hline Female (\%) & $18(50.0 \%)$ & $28(80 \%)$ & 0.002 & $10(92 \%)$ & 0.02 \\
\hline Age (mean $\pm S D$, range) & $50 \pm 17.7(14-84)$ & $57 \pm 13.9(24-80)$ & 0.23 & $62 \pm 8.1(51-80)$ & 0.07 \\
\hline Obesity (\%) & $14(41 \%)$ & $19(54 \%)$ & 0.20 & $5(45 \%)$ & 0.70 \\
\hline Diabetes mellitus (\%) & $8(22 \%)$ & $10(28 \%)$ & 0.54 & $4(36 \%)$ & 0.35 \\
\hline Hyperlipidemia (\%) & $5(14 \%)$ & $11(31 \%)$ & 0.08 & $3(27 \%)$ & 0.93 \\
\hline AST (U/L) (mean $\pm S D$, range) & $81 \pm 40.0(14-193)$ & $106 \pm 90.2(29-532)$ & 0.20 & $104 \pm 48.2(56-177)$ & 0.15 \\
\hline ALT (U/L) (mean $\pm S D$, range) & $114 \pm 73.7(23-312)$ & $142 \pm 104.1(27-524)$ & 0.26 & $136 \pm 75.4(25-282)$ & 0.24 \\
\hline ALP (U/L) (mean $\pm S D$, range) & $302 \pm 107.4(133-529)$ & $300 \pm 123.8(71-636)$ & 0.95 & $278 \pm 71.8(217-436)$ & 0.58 \\
\hline$\gamma$-GTP $(\mathrm{U} / \mathrm{L})($ mean $\pm \mathrm{SD}$, range $)$ & $187 \pm 402.3(14-2110)$ & $124 \pm 141.1(38-650)$ & 0.72 & $78.5 \pm 51.1(52-153)$ & 0.83 \\
\hline $\operatorname{IgG}(\mathrm{mg} / \mathrm{dl})$ & $1387.7 \pm 354.9$ & $1426.3 \pm 397.5$ & 0.79 & $1766.43 \pm 471.5$ & 0.053 \\
\hline AIH score $<10$ & $32(97 \%)$ & $16(53 \%)$ & 0.0000005 & $3(33 \%)$ & 0.00003 \\
\hline 10-15 (Probable AIH) & $1(3 \%)$ & $14(47 \%)$ & & $6(67 \%)$ & \\
\hline $15<$ (Definite AIH) & 0 & 0 & & 0 & \\
\hline
\end{tabular}

ANA, antinuclear antibodies; NASH, non-alcoholic steatohepatitis; *, p value versus ANA-negative NASH 
Table 2 Histological features in the patients with non-alcoholic steatohepatitis with and without serum antinuclear antibody

\begin{tabular}{|c|c|c|c|c|c|c|}
\hline & score & $\begin{array}{c}\text { ANA-negative } \\
\text { NASH } \\
(n=36)\end{array}$ & $\begin{array}{c}\text { ANA-positive } \\
\text { NASH } \\
(n=35)\end{array}$ & p value* & $\begin{array}{c}\text { gh-titer AN } \\
\text { NASH } \\
(n=11)\end{array}$ & p value* \\
\hline \multirow[t]{3}{*}{ Grade } & 1 & $18(50 \%)$ & $11(31 \%)$ & 0.054 & $2(18 \%)$ & 0.02 \\
\hline & 2 & 17 (47\%) & 19 (54\%) & & $6(55 \%)$ & \\
\hline & 3 & $1(3 \%)$ & $5(15 \%)$ & & $3(27 \%)$ & \\
\hline \multirow[t]{4}{*}{ Stage } & 1 & 17 (47\%) & $15(43 \%)$ & 0.46 & $1(9 \%)$ & 0.009 \\
\hline & 2 & $11(31 \%)$ & $8(23 \%)$ & & $3(27 \%)$ & \\
\hline & 3 & 7 (19\%) & $11(31 \%)$ & & 7 (64\%) & \\
\hline & 4 & $1(3 \%)$ & $1(3 \%)$ & & 0 & \\
\hline \multirow[t]{3}{*}{ Portal inflammation } & 1 & $22(61 \%)$ & $14(40 \%)$ & 0.039 & $1(9 \%)$ & 0.001 \\
\hline & 2 & $9(25 \%)$ & $9(26 \%)$ & & $4(36 \%)$ & \\
\hline & 3 & 5 (14\%) & $12(34 \%)$ & & $6(55 \%)$ & \\
\hline \multirow[t]{4}{*}{ Interface activity } & 0 & $22(61 \%)$ & $15(43 \%)$ & 0.036 & 0 & 0.00005 \\
\hline & 1 & $10(27 \%)$ & $6(17 \%)$ & & $3(27 \%)$ & \\
\hline & 2 & $2(6 \%)$ & $10(29 \%)$ & & $6(55 \%)$ & \\
\hline & 3 & $2(6 \%)$ & $4(11 \%)$ & & $2(18 \%)$ & \\
\hline \multirow[t]{3}{*}{ Lobular inflammation } & 1 & $19(53 \%)$ & $21(60 \%)$ & 0.74 & $6(55 \%)$ & 0.63 \\
\hline & 2 & $15(41 \%)$ & $10(29 \%)$ & & $2(18 \%)$ & \\
\hline & 3 & $2(6 \%)$ & $4(11 \%)$ & & $3(27 \%)$ & \\
\hline
\end{tabular}

(Cont'd) 


\begin{tabular}{|c|c|c|c|c|c|c|}
\hline \multirow[t]{3}{*}{ Steatosis } & 1 & $17(47 \%)$ & $21(60 \%)$ & \multirow[t]{2}{*}{0.33} & $10(91 \%)$ & \multirow[t]{3}{*}{0.01} \\
\hline & 2 & $10(27 \%)$ & $7(20 \%)$ & & $1(9 \%)$ & \\
\hline & 3 & $9(25 \%)$ & $7(20 \%)$ & & 0 & \\
\hline \multirow[t]{3}{*}{ Hepatocellular ballooning } & 1 & $17(47 \%)$ & $6(17 \%)$ & \multirow[t]{3}{*}{0.0008} & $2(18 \%)$ & \multirow[t]{3}{*}{0.06} \\
\hline & 2 & $11(31 \%)$ & $8(23 \%)$ & & $4(36 \%)$ & \\
\hline & 3 & $8(22 \%)$ & $21(60 \%)$ & & $5(45 \%)$ & \\
\hline Mallory body (\%) & & $10(28 \%)$ & $15(43 \%)$ & 0.28 & $6(55 \%)$ & 0.10 \\
\hline Cytoplasmic coagulum (\%) & & $21(58 \%)$ & 24 (69\%) & 0.37 & $8(73 \%)$ & 0.39 \\
\hline
\end{tabular}

ANA, antinuclear antibodies; NASH, non-alcoholic steatohepatitis; High-titer ANA, the titer of ANA was 320-fold or more; *, p value versus ANA-negative NASH; 
Table 3 Inflammatory cell profiles in portal area in the patients with NASH with and without serum antinuclear antibody

\begin{tabular}{lcccc}
\hline & $\begin{array}{c}\text { ANA negative NASH } \\
\mathrm{n}=8\end{array}$ & $\begin{array}{c}\text { ANA positive NASH } \\
\mathrm{n}=8\end{array}$ & p value* $^{*}$ & $\begin{array}{c}\text { AIH } \\
\mathrm{n}=10\end{array}$ \\
\hline CD20/CD3 (ratio) & $0.16 \pm 0.11^{\mathrm{a}}$ & $0.17 \pm 0.10^{\mathrm{a}}$ & 0.83 & $0.34 \pm 0.17$ \\
CD8/CD3 (ratio) & $0.61 \pm 0.07^{\mathrm{b}}$ & $0.56 \pm 0 / 12$ & 0.37 & $0.46 \pm 0.10$ \\
CD138/CD3 (ratio) & $0.06 \pm 0.06^{\mathrm{b}}$ & $0.05 \pm 0.06^{\mathrm{b}}$ & 0.83 & $0.26 \pm 0.06$ \\
\hline
\end{tabular}

ANA, antinuclear antibodies; NASH, non-alcoholic steatohepatitis; *, p value versus ANA-negative NASH; a, p<0.05 vs AIH; b, p<0.01 vs AIH 
Table 4 Inflammatory cell profiles in hepatic lobules in the patients with NASH with and without serum antinuclear antibody

\begin{tabular}{|c|c|c|c|c|}
\hline & $\begin{array}{c}\text { ANA positive NASH } \\
n=15\end{array}$ & $\begin{array}{l}\text { ANA negative NASH } \\
n=15\end{array}$ & p value* & $\begin{array}{l}\mathrm{AIH} \\
\mathrm{n}=10\end{array}$ \\
\hline CD20/CD3 (ratio) & $0.097 \pm 0.06$ & $0.097 \pm 0.04$ & 0.83 & $0.10 \pm 0.07$ \\
\hline CD8/CD3 (ratio) & $0.60 \pm 0.11$ & $0.55 \pm 0.07$ & 0.14 & $0.54 \pm 0.07$ \\
\hline MPO/CD3 (ratio) & $0.19 \pm 0.12^{\mathrm{a}}$ & $0.16 \pm 0.08^{\mathrm{a}}$ & 0.43 & $0.01 \pm 0.02$ \\
\hline CD138/CD3 (ratio) & $0.013 \pm 0.015^{\mathrm{a}}$ & $0.018 \pm 0.018^{\mathrm{a}}$ & 0.45 & $0.17 \pm 0.08$ \\
\hline
\end{tabular}

ANA, antinuclear antibodies; NASH, non-alcoholic steatohepatitis; *, p value versus ANA-negative NASH; a, p<0.01 vs AIH 
Table 5 Semi-quantitative assessment of 8-OHdG, 4-HNE and 4-HHE in the patients with NASH with and without serum antinuclear antibody

\begin{tabular}{|c|c|c|c|c|}
\hline & & ANA negative NASH & ANA positive NASH & \\
\hline & score & & & p value \\
\hline 8-OHdG positive rate $(\%)$ & & $39.7 \pm 31.0$ & $30.2 \pm 25.0$ & 0.17 \\
\hline 4-HNE positive rate (\%) & & $20.1 \pm 14.8$ & $20.2 \pm 13.8$ & 0.99 \\
\hline \multirow[t]{4}{*}{ 4-HHE } & 0 & 7 & 8 & 0.94 \\
\hline & 1 & 11 & 7 & \\
\hline & 2 & 4 & 4 & \\
\hline & 3 & 4 & 5 & \\
\hline
\end{tabular}

ANA, antinuclear antibodies; NASH, non-alcoholic steatohepatitis; 8-OHdG, 8-hydroxydeoxyguanosine; 4-HNE, 4-hydroxy-2'-nonenal; 4-HHE, 4-hydroxy- 2-hexenal. 


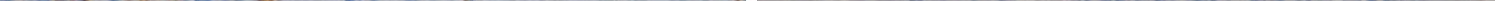




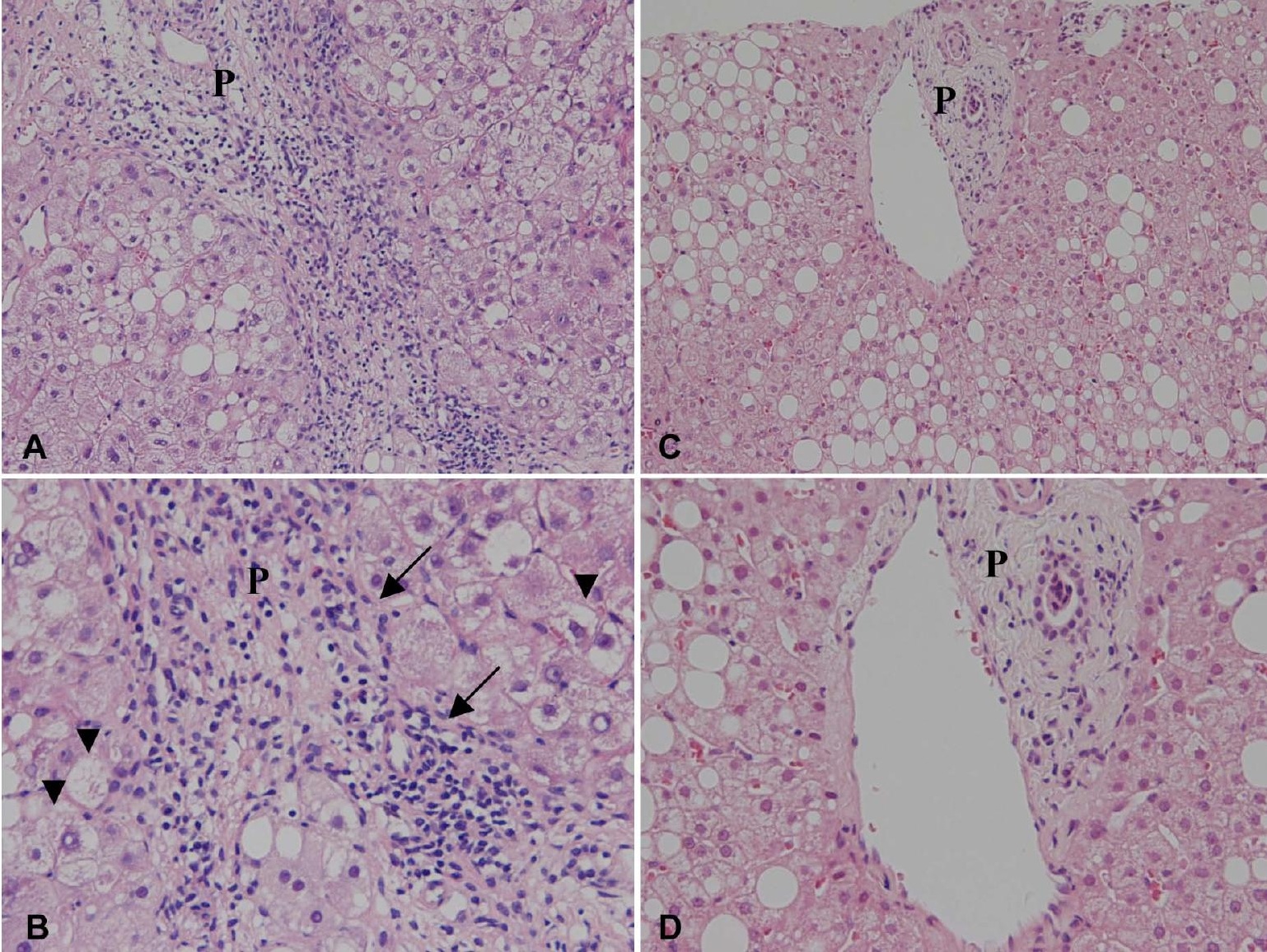


